\title{
THE ADDITIVE STRUCTURE OF MODELS OF ARITHMETIC
}

\author{
LEONARD LIPSHITZ AND MARK NADEL
}

\begin{abstract}
It is shown that for a model of Presburger arithmetic to have an expansion to a model of Peano arithmetic it is necessary that the model be recursively saturated. For countable models this condition is also sufficient; for uncountable models it is not.
\end{abstract}

This note arises from consideration of the question of when a model of Presburger arithmetic (Pr) (see the definition below) can be expanded to a model of Peano arithmetic (P). In the countable case an answer is given by the following:

TheOReM 1. A countable, nonstandard model $\langle A,+\rangle$ of Presburger arithmetic can be expanded to a model $\langle A,+, \cdot\rangle$ of Peano arithmetic if and only if it is recursively saturated.

In [3] it is shown that the recursively saturated models of $P$ are just the nonstandard models of $P$ which can be expanded to models of a certain fragment of analysis. Theorem 1 again involves the application of the abstract characterization of recursive saturation to an even more familiar algebraic setting. Theorem 1 is proved in $\S 1$. The analogy, however, between this result and that of [3] does not go any further. While the main result of [3] holds without modification for all infinite cardinalities, Theorem 1 does not extend as shown by the example of $\$ 2$ below. The complete generality of the main result of [3] is easy to understand in view of the simple expansion which is given in a uniform way. The expansion provided for by Theorem 1 is obtained in a completely nonconstructive fashion from the general fact that countable recursively saturated models are resplendent [2]. This nonconstructivity can be explained, at least in part, by Theorem 2 of $\$ 3$.

Definitions. (1) Presburger arithmetic ( $\mathrm{Pr})$ is the theory of the additive semigroup $\langle\mathbf{N},+\rangle$. We shall think of $\operatorname{Pr}$ in the language $\left\langle 0,1,+,\left\langle, \equiv_{n}\right\rangle_{n=1,2,3, \ldots}\right.$, since in this language Pr admits an elimination of quantifiers [6]. $<$ and $\equiv_{n}$ are definable from 0,1 , and + . A complete set of axioms for $\mathrm{Pr}$ is:

(i) The axioms for discretely ordered abelian semigroups with 0 , and smallest nonzero element 1 .

(ii) $x<y \rightarrow x+z<y+z$. 
(iii) $\forall x \exists y(x=n y \vee x=n y+1 \vee \cdots \vee x=n y+n-1)$.

(iv) ${ }_{n} x \equiv_{n} y \leftrightarrow \exists z(x=y+n z \vee y=x+n z)$.

$\operatorname{Pr}^{\prime}$ will denote the theory of discretely ordered abelian groups with smallest positive element 1, satisfying (ii), (iii) ${ }_{n}$ and (iv) ${ }_{n}$. Given a model of Pr we can define a model of $\mathrm{Pr}^{\prime}$ as equivalence classes of ordered pairs (in the same way that we obtain $\mathbf{Z}$ from $\mathbf{N}$ ) and given a model of $\mathrm{Pr}^{\prime}$, we can recover the model of $\operatorname{Pr}$ as the set of elements $\geqslant 0$.

(2) A model $\mathfrak{A}$ is called recursively saturated if for each recursive set of formulas $\Phi(x, \bar{y})$ in the variables $x, \bar{y}$, the following is true in $\mathfrak{A}$ :

$$
\forall \bar{y}\left[\left(\widehat{\Phi}_{\Phi^{\prime} \subseteq \Phi, \Phi_{\text {finite }}} \exists x \widehat{\varphi}_{\varphi \in \Phi^{\prime}} \varphi(x, \bar{y})\right) \rightarrow \exists x \widehat{\varphi}_{\varphi \Phi} \varphi(x, \bar{y})\right] \text {. }
$$

1. Proof of Theorem 1. Let $\varphi_{i}$ denote the formula with Gödel number $i$.

LEMMA 1. Let $\Gamma=\left\{\varphi_{i}(x, \bar{y}): i \in R\right\}$ be a recursive type in the language of Pr. Then there is a formula $\psi(z, x, \bar{y})$ in $\langle+, \cdot, 0,1\rangle$ such that for each $i \in R$, $\mathrm{P} \vdash\left[\psi(\underline{i}, x, \bar{y}) \leftrightarrow \varphi_{i}(x, \bar{y})\right]$, and for each $i \notin R, \mathrm{P} \vdash \psi(\underline{i}, x, \bar{y})$.

Proof. Let $A(n)$ represent the recursive relation $R$, so $\mathrm{P} \vdash A(\underline{n})$ if $n \in R$ and $\mathrm{P} \vdash \neg A(\underline{n})$ if $n \notin R$. From the elimination of quantifiers for $\operatorname{Pr}$ [6], there is a primitive recursive function $f$, such that if $\varphi_{n}$ is a formula in the language of $\operatorname{Pr}$ then $\varphi_{f(n)}$ is a quantifier free formula in the language $\left\langle 0,1,+,\left\langle, \equiv_{n}\right\rangle_{n=1,2,3, \ldots}\right.$, which is equivalent to $\varphi_{n}$ and has the same free variables as $\varphi_{n}$. There is also a primitive recursive function $g$ such that if $\varphi_{n}$ is a quantifier free formula in $\left\langle 1,+,\left\langle, \equiv_{k}\right\rangle\right.$, then $\varphi_{g(n)}$ is an equivalent existential formula in $\langle+, \cdot, 0,1\rangle$, such that $P \vdash \varphi_{n} \leftrightarrow \varphi_{g(n)}$. Let $B(u, v)$ represent the recursive relation $g(f(w))=v$. Let $\Sigma \operatorname{Sat}(i, x, \bar{y})$ be a formula which states that $i$ is the Gödel number of an existential formula $\varphi_{i}(\bar{u}, \bar{v}$ ) (in $+, \cdot, 0,1)$ and $x, \bar{y}$ satisfy $\varphi_{i}(\bar{u}, \bar{v})$. This is easy to write down. Then $\mathrm{P}-$ $\varphi_{i}(x, \bar{y}) \leftrightarrow \Sigma \operatorname{Sat}(\underline{i}, x, \bar{y})$. Let $\psi(z, x, \bar{y})$ be $\{A(z) \wedge \exists w[B(z, w) \wedge$ $\Sigma \operatorname{Sat}(w, x, \bar{y})]\} \vee \neg A(z)$.

LEMMA 2. Suppose that $\langle A,+, \cdot\rangle$ is a nonstandard model of $\mathrm{P}$. Then $(A,+)$ is recursively saturated.

Proof. Let $\Gamma=\left\{\varphi_{i}(x, \bar{y}): i \in R\right\}$ be a recursive type in the language of $\operatorname{Pr}$ over $\langle A,+\rangle$ and let $\bar{a} \in \bar{A}$ be such that $\langle A,+\rangle \vDash \exists x \bigwedge_{i<n, i \in R} \varphi_{i}(x, \bar{a})$ for all $n \in \mathbf{N}$. Let $\psi(i, x, \bar{y})$ be as given by Lemma 1 . Then, for each $n \in \omega$,

$$
\langle A,+, \cdot\rangle \vDash \exists x \forall i \leqslant n \psi(i, x, \bar{a}) .
$$

Hence, for some nonstandard $\alpha_{0} \in A$,

$$
\langle A,+, \cdot\rangle \vDash \exists x \forall i \leqslant \alpha_{0} \psi(i, x, \bar{a}) .
$$

Consequently, for some $b \in A$,

$$
\langle A,+, \cdot\rangle \vDash \forall i \leqslant \alpha_{0} \psi(i, b, \bar{a}) .
$$

In particular then, for each $n \in \omega$,

$$
\langle A,+, \cdot\rangle \vDash \psi(\underline{n}, b, \bar{a}),
$$


whence

$$
\langle A,+\rangle \vDash \varphi_{n}(b, \bar{a}) .
$$

Thus

$$
\langle A,+\rangle \vDash \bigwedge_{n<\omega} \varphi_{n}(b, \bar{a}) \text {. }
$$

The following lemma is a special case of 2.3(ii) of [2].

LEMMA 3. If $\mathfrak{A}$ is a countable recursively saturated model of $\operatorname{Pr}$ then $\mathfrak{A}$ can be expanded to a model of $\mathrm{P}$.

Theorem 1 now follows immediately from Lemmas 2 and 3.

REMARKS. (i) The corresponding theorem holds with multiplication in place of addition. The elimination of quantifiers for $\langle\mathbf{N}, \cdot\rangle$ follows from that for $\langle\mathbf{N},+\rangle$ and the theorems of [4].

2. In this section we shall give an example of an uncountable $\aleph_{0}$-saturated model of Pr which cannot be expanded to a model of P. It will be convenient to work with models of $\mathrm{Pr}^{\prime}$.

Let $\mathbf{Z}^{*}$ be an $\aleph_{1}$-saturated model of $\operatorname{Pr}^{\prime}$ of cardinality $>2^{\kappa_{0}}$ and let $Q^{*}$ be an $\kappa_{1}$-saturated model of $\mathrm{Th}(\mathbf{Q},+,<)$ (i.e. the theory of infinitely divisible ordered abelian groups) of cardinality $2^{\aleph_{0}}$. Let $\mathfrak{A}=Q^{*} \times \mathbf{Z}^{*}$ with addition defined coordinatewise and with the lexicographic ordering $\left(q_{1}, z_{1}\right)<\left(q_{2}, z_{2}\right)$ if and only if $q_{1}<q_{2}$ or $q_{1}=q_{2}$ and $z_{1}<z_{2}$. It is clear that $\mathfrak{A}$ satisfies the axioms of $\operatorname{Pr}^{\prime}$. We shall show

(1) There is no multiplication, ', on $\mathfrak{A}$ satisfying the rules $x<y \rightarrow x z<y z$, $(x \pm y) z=x z \pm y z$ and $1 \cdot z=z$.

(2) $\mathfrak{A}$ is an $\aleph_{0}$-saturated model of $\mathrm{Pr}^{\prime}$.

If we let $\mathfrak{A}_{0}$ be the elements of $\mathfrak{A}$ which are $\geqslant(0,0)$ then $\mathfrak{A}_{0}$ is the required model of $\operatorname{Pr}$.

Proof of (1). Suppose that such a multiplication exists. Let $a=(q, 0)$, $q>0\left(q \in Q^{*}\right)$. Then $a>(0, z)$ for all $z \in \mathbf{Z}^{*}$. Let $\mathfrak{A} / \mathbf{Z}^{*}$ denote the factor group (as an ordered group) and denote the coset $y+Z^{*}$ by $[y]$. Consider the mapping $\mathfrak{A} \rightarrow \mathfrak{A} / \mathbf{Z}^{*}$ defined as follows: $x \rightarrow[x a]$. This is a $1-1$ order preserving homomorphism of $\mathfrak{A}$ into $\mathfrak{A} / \mathbf{Z}^{*} \simeq Q^{*}$ since if $x_{1}>x_{2}$ then $\left(x_{1}-x_{2}\right) a$ $\geqslant 1 \cdot a=a>(0, z)$ for all $z \in \mathbf{Z}^{*}$ and, hence, $\left[x_{1} a\right] \neq\left[x_{2} a\right]$. But $\overline{\overline{\mathfrak{A}}}>2^{\aleph_{0}}$ and $\overline{\bar{Q}}^{*}=2^{\kappa_{0}}$.

Proof of (2). Let $\Sigma$ be a type over the constants $\bar{a}=\left(a_{1}, \ldots, a_{n}\right)$ which is finitely satisfiable in $\mathfrak{A}$. Let $\Sigma_{0} \supseteq \Sigma$ be a complete type over $\bar{a}$ in $\operatorname{Th}(\mathfrak{A}, \bar{a})$. Since $\operatorname{Th}(\mathfrak{A}, \bar{a})$ is complete, $\Sigma_{0}$ is finitely satisfiable over $\bar{a}$. Using the elimination of quantifiers for $\operatorname{Pr}\left(\right.$ or $\operatorname{Pr}^{\prime}$ ) we can replace each formula of $\Sigma_{0}$ by a disjunction of conjunctions of atomic formulas in $\left\langle 0,1,+,\left\langle, \equiv_{n}\right\rangle\right.$. (Notice that the negation of an atomic formula is equivalent to a finite disjunction of atomic formulas.) Since $\Sigma_{0}$ is maximal we have that $\Sigma_{0}$ is equivalent to $\Sigma_{1} \cup \Sigma_{2} \cup \Sigma_{3}$, where $\Sigma_{1}$ is a set of formulas of the form $x \equiv_{n} i_{n}$ and $\Sigma_{2}$ is a set of formulas of the form $k_{n} x \gtrless t_{n}(\bar{a}), k_{n} \in \mathrm{N}$, where $t(\bar{a})$ is a 
variable free term, and $\Sigma_{3}$ is a set of equalities. We can assume that $\Sigma_{3}$ is empty, since otherwise the type is principal. Let $t_{n}(\bar{a})=k_{n} b_{n}+j_{n}, 0 \leqslant j_{n}<$ $k_{n}$. Then $k_{n} x \gtrless t_{n}(a) \leftrightarrow x \gtrless b_{n}$. Let $\Sigma_{2}^{\prime}$ be the set of formulas of this form corresponding to the formula of $\Sigma_{2}$. Then $\Sigma_{0}$ is equivalent to $\Sigma_{1} \cup \Sigma_{2}^{\prime}$. Let $\bar{\Sigma}_{2}=\left\{x \gtrless[c] \mid x \gtrless c \in \Sigma_{2}^{\prime}\right\}$. Hence $\bar{\Sigma}_{2}$ is a type over $\mathfrak{A} / \mathbf{Z}^{*} \simeq Q^{*}$. If $\bar{\Sigma}_{2}$ is consistent then there is a $q \in Q^{*}$ such that $q$ satisfies $\bar{\Sigma}_{2}$. Then all the inequalities in $\Sigma_{2}^{\prime}$ are satisfied by $(q, z)$ for any $z \in \mathbf{Z}^{*}$. Choose $z_{0} \in \mathbf{Z}^{*}$ satisfying $\Sigma_{1}$. Then $\left(q, z_{0}\right)$ satisfies $\Sigma$. If $\bar{\Sigma}_{2}$ is inconsistent then there are two inequalities $b_{1}<x<b_{2}$ in $\Sigma_{2}$ with $\left[b_{1}\right]=\left[b_{2}\right]=[(q, 0)]$, say. Let $\bar{\Sigma}_{2}=\{x \gtrless$ $\left.z_{n} \mid x \gtrless\left(q, z_{n}\right) \in \Sigma_{2}\right\}$. Think of $\Sigma_{1} \cup \bar{\Sigma}_{2}$ as a type over $\mathbf{Z}^{*}$. It is finitely satisfiable. Hence by the saturation of $\mathbf{Z}^{*}$ we can choose $z \in \mathbf{Z}^{*}$ which satisfies it. Then $(q, z)$ satisfies $\Sigma_{1} \cup \Sigma_{2}^{\prime}$ in $\mathfrak{A}$.

REMARK. Let $E$ be the class of models of Pr which can be expanded to models of P. Let $R$ be the class of rec. saturated models of Pr. $E$ is clearly $\Sigma$ definable in set theory, while $R$ is $\Pi$ (actually $\Delta$ ) definable. We know from Theorem 1 and the above example that $E \cap H C=R \cap H C$ and $E \subset R$, where $H C$ is the set of hereditarily countable sets. Now, by Lévy's absoluteness theorem, if $E$ were $\Pi$ definable, then $E$ would have to equal $R$. Therefore $E$ is not $\Pi$ definable. Perhaps, the search for a condition equivalent to membership in $E$ might give rise to notions of some more general model theoretic interest.

3. It is easy to see that the order type of any nonstandard model $\mathfrak{A}$ of $\mathbf{P}$ (or even $\operatorname{Pr}$ ) is of the form $\omega+\mathbf{Z} \cdot \rho$, where $\rho$ is dense without endpoints. It is also fairly well known that if $\mathfrak{A}$ is a model of $\mathrm{P}$ then $\rho$ cannot be the order type of the reals, $\mathbf{R}$. On the other hand $\omega+\mathbf{Z} \cdot \mathbf{R}$ is a model of $\operatorname{Pr}$, with addition interpreted in the obvious way. Even though the recursive saturation of an arbitrary model of Pr does not guarantee the existence of an expansion to $P$, we show below that it will prevent $\rho$ from being $\mathbf{R}$.

Proposition 1. Suppose that $\mathfrak{A}$ is a recursively saturated model of Pr. Then $\mathfrak{A}$ does not have order type $\omega+\mathbf{Z} \cdot \mathbf{R}$.

Proof. We assume that the order type of $(A-\omega) / Z$ is sequentially complete and show that $\mathfrak{A}$ is not recursively saturated. Let $a$ be a nonstandard element of $A$. Consider the set $\{n a: n \in \omega\}$. For $m \neq n, m a$ and $n a$ are in different Z-blocks of $A$. Suppose that $b$ is in the least Z-block greater than $n a$ for all $n \in \omega$. Consider the recursive type $\Phi(x)=\{n a<x, x+n<b: n$ $\in \omega\} . \Phi(x)$ is clearly finitely satisfiable but not satisfiable by the choice of the Z-block of $b$.

The reason that recursive saturation is sufficient for the above result seems to be that the order type of $\mathbf{R}$ can be excluded using a countable sequence. In contrast we next give a stronger result for $\mathrm{P}$ in which uncountability plays an essential role and which can fail for recursively saturated models of $\mathrm{Pr}$.

Proposition 2. Let $\mathfrak{A}$ be an uncountable model of $\mathrm{P}$ and let $\mathrm{G}$ be a bounded, additive subsemigroup of $\mathfrak{A}$ such that whenever $x<y \in G, x \in G$. Then the 
order type of $(\mathfrak{A}-\omega) / G$ cannot be embedded in $\mathbf{R}$.

Proof. Let $a$ be an upperbound for $G$. Consider the mapping $x \rightarrow$ ([xa], $[(x+1) a])$ of elements of $\mathfrak{A}$ to open intervals in $(\mathfrak{A}-\omega) / G$. Since $a$ is larger than every element of $G$ this mapping is one-to-one and the intervals corresponding to different $x$ 's are disjoint. But there is no such uncountable set of disjoint open intervals in $\mathbf{R}$.

REMARK. From the counterexample of $\S 2$ and Keisler's extension to countable fragments of $L_{\omega_{1} \omega}$ of Vaught's two cardinal theorem [5, Theorem 30], we deduce the existence of an uncountable recursively saturated model $\mathfrak{A}$ of $\mathrm{Pr}$ with a bounded subsemigroup $G$ such that $(\mathfrak{A}-\omega) / G$ is countable (and hence order embeddable in $\mathbf{R}$ ) as follows. For convenience we work with $\mathrm{Pr}^{\prime}$. We use the language of $\operatorname{Pr}^{\prime}$ with two new unary predicates $Z$ and $Q$ which will serve to pick out the $Z^{*}$ and $Q^{*}$ of the example of $\S 2$.

Consider the infinitary theory $T$ whose axioms are:

(1) $\mathrm{Pr}^{\prime}$.

(2) Sentences in the language of $\operatorname{Pr}^{\prime}$ which guarantee recursive saturation for models of $\mathrm{Pr}^{\prime}$.

(3) $\operatorname{Pr}^{\prime}(Z)$ (the axioms of $\operatorname{Pr}^{\prime}$ relativated to $Z$ ).

(4) Sentences saying that each coset of the group factored by $Z$ contains exactly one element of $Q$.

(5) $\exists x[Q(x) \wedge \forall z(Z(z) \rightarrow z<x)]$.

It is clear that $T$ is a theory in some countable fragment of $L_{\omega, \omega}$. Let $\mathfrak{A}=\mathbf{Z}^{*} \times Q^{*}$ as in $\S 2$. Without difficulty we can arrange that $\overline{\bar{Z}}^{*}=2^{2^{\omega_{0}}}$ and $\bar{Q}^{*}=2^{\kappa_{0}} .\left\langle A,+,<, Z^{*} \times\{0\},\{0\} \times Q^{*}\right\rangle$ is a model of $T$ of type $\left(2^{2^{\kappa_{0}}}, 2^{\kappa_{0}}\right)$, where the second predicate is taken to be $Q$. By the Vaught-Keisler two cardinal theorem, $T$ has a model $\left\langle B,+,\left\langle, Z^{\prime}, Q^{\prime}\right\rangle\right.$ of type $\left(\aleph_{1}, \aleph_{0}\right)$. By (5) and (3) $Z^{\prime}$ is a bounded subgroup of $B$ and by (4) $B / Z^{\prime}$ is countable, since $Q^{\prime}$ is.

Since $\operatorname{Pr}$ is decidable but $\mathrm{P}$ is not, multiplication cannot be first order definable from addition. However, even more is true.

TheOREM 2. Let $\mathfrak{A}=\langle A,+\rangle$ be a nonstandard model of Pr. There is no relation $\cdot \in \mathrm{HYP}_{\mathfrak{A}}$ such that $\langle A,+, \cdot\rangle$ is a model of $\mathrm{P}$.

Proof. Assume there is a relation $\cdot \in \mathrm{HYP}_{\mathfrak{A}}$ such that $\langle A,+, \cdot\rangle$ is a model of $\mathrm{P}$. Then, by previous results, $\langle A,+\rangle$ is recursively saturated, whence, by [1], every subset of $A$ in $\mathrm{HYP}_{\mathfrak{A}}$ is first order definable over $\mathfrak{A}$. We now obtain the nonfirst order definability of multiplication without appealing to the undecidability of P. Suppose the ternary relation $x \cdot y=z$ is defined by $\varphi(x, y, z)$.

Let $T=\operatorname{Th}\langle A,+, \cdot\rangle$. Since $\mathrm{P}$ is not complete, for some sentence $\theta \in T$, $T^{\prime}=\mathrm{P}+\neg \theta$ is a consistent recursive theory (extending the complete theory Pr).

It is enough to show the theorem for countable $\mathfrak{A}$ since the general case would follow from Lévy absoluteness. So let us assume $\mathfrak{A}$ is countable. 
Since $\mathfrak{A}$ is countable and recursively saturated there is a relation $\times$ such

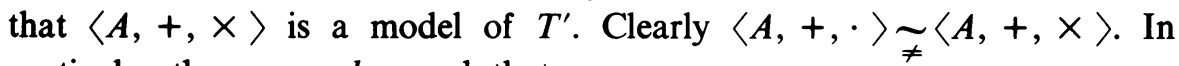
particular, there are $a, b, c$ such that

$$
\langle A,+, \times\rangle \vDash a \times b=c \text { but }\langle A+, \times\rangle \vDash \neg \varphi(a, b, c) .
$$

Now, using induction in $\langle A+, \times\rangle$ let

$$
\begin{aligned}
a & =\mu x \exists y \exists z[x \times y=z \wedge \neg \varphi(x, y, z)], \\
b & =\mu y \exists z[a \times y=z \wedge \neg \varphi(a, y, z)] .
\end{aligned}
$$

Since clearly $b \neq 0$ we may assume $b-1$ exists. But then,

$$
a \cdot(b-1)=a \times(b-1) .
$$

Now, using the fact that both $\langle A,+, \cdot\rangle$ and $\langle A,+, \times\rangle$ are models of $\mathrm{P}$ we have

$$
a \cdot b=a \cdot(b-1)+a=a \times(b-1)+a=a \times b,
$$

a contradiction.

COROLlaRY. Suppose $\langle A,+\rangle$ is a countable nonstandard model of $\operatorname{Pr}$, then there is no relation $\cdot, \Delta_{1}^{1}$ definable over $\langle A,+\rangle$ such that $\langle A,+, \cdot\rangle$ is a model of $\mathbf{P}$.

\section{REFERENCES}

1. K. J. Barwise, Admissible sets and structures, Springer-Verlag, Berlin and New York, 1975.

2. K. J. Barwise and John Schlipf, An introduction to recursively saturated and resplendent models, J. Symbolic Logic 41 (1976), 531-536.

3. On recursively saturated models of arithmetic, Lecture Notes in Math., no. 498, Springer-Verlag, Berlin, 1975.

4. Solomon Feferman and R. L. Vaught, The first order properties of products of algebraic systems, Fund. Math. 47 (1959), 57-103.

5. M. Presburger, Über die Vollständigkeit eines gewissen Systems der Arithmetik ganzer Zahlen, in welchem die Addition als einzige Operation hervortritt, Comptes-rendus du I congrès des mathématiciens der pays slaves, Warsaw, 1929, pp. 92-101.

6. H. J. Keisler, Model theory for infinitary logic, North-Holland, Amsterdam, 1971.

School of Mathematics, The Institute for Advanced Study, Princeton, New Jersey 08540

Department of Mathematics, Purdue University, West Lafayette, Indiana 47907

Department of Mathematics, University of Notre Dame, Notre Dame, Indiana 46556 